\title{
Género y niveles de adherencia al tratamiento antihipertensivo en el personal de la Universidad Estatal a Distancia de Costa Rica
}

\author{
Lourdes Arce Espinoza' y Julián Monge-Nájera² \\ 'Servicio Médico, Universidad Estatal a Distancia, 474-2050 San Pedro Montes de Oca, San José, Costa Rica; larce@uned.ac.cr \\ 2 Vicerrectoría de Investigación, Universidad Estatal a Distancia, 474-2050 San Pedro Montes de Oca, San José,Costa Rica; julianmonge@gmail.com \\ Recibido 20-VII-2009 Corregido 3-IX-2009 Aceptado 11-IX-2009
}

\begin{abstract}
Gender and levels of adherence to antihypertensive treatment in personnel of the Costa Rican Distance Education University. Among chronic diseases, hypertension is one of the top causes of morbidity and mortality worldwide. Until now, health efforts have focused on decreasing this condition but the treatments have been ineffective and costly, and hypertension is one the least controlled diseases. Several studies have found that adherence to treatment in chronic diseases define therapeutic success. In turn, adherence depends on several factors from both the patients and their environment, but the relationship with the physician seems to be the main factor. In this context, gender is also an important factor in how patients deal with their disease. For this study, we interviewed 39 women and 41 men with hypertension who visited the health department at the Costa Rican Distance Education University, UNED, between January $14^{\text {th }}, 2002$ and December $15^{\text {th }}, 2008$. We wanted to see if there were gender differences in their relationship with the physician and their adherence to treatment. Statistical analysis found no significant gender differences in personal characteristics of patients or their environment. Gender differences in their relationship with the physicians are also minimal and in general both the relationship with the physician as the adhesion to hypertension treatment are good throughout this population.
\end{abstract}

\section{KEY WORDS}

Hypertension, antihypertensive treatment, epidemiology, mortality, gender, cardiovascular risk

\section{RESUMEN}

La hipertensión arterial es una de las enfermedades crónicas que ocupa los primeros lugares de morbi-mortalidad a nivel mundial. Hasta ahora, las acciones de salud se han enfocado en la disminución de esta patología; sin embargo los tratamientos han sido poco efectivos y los costos elevados, y la hipertensión continua siendo una de las enfermedades con menos control. Diversos estudios han determinado que la adherencia al tratamiento en enfermedades crónicas define el éxito terapéutico. La adherencia a su vez, depende de varios factores tanto del paciente como de su entorno, siendo clave la relación con el médico. En este contexto, el género (femenino o masculino) es un factor importante. Para este estudio se entrevistaron 39 mujeres y 41 hombres hipertensos que visitaron el consultorio médico de la Universidad Estatal a Distancia entre el 14 de enero de 2002 y el 15 de diciembre de 2008, para buscar diferencias por género en los pacientes, su relación con el personal médico y su adherencia al tratamiento. El análisis estadístico indicó que no hay diferencias de importancia debidas al género en las características físicas y personales de los pacientes, ni en su ambiente. Las diferencias de género en cuanto a la relación con el médico son mínimas y en general tanto la relación como la adherencia son buenas en toda esta población.

\section{PALABRAS CLAVE}

Hipertensión, tratamiento antihipertensivo, mortalidad, género, epidemiología, riesgo cardiovascular
Las causas de morbilidad y mortalidad están en transición a nivel mundial, pasando de las enfermedades transmisibles a las no transmisibles $y$ crónicas, como las enfermedades cardiovasculares (Organización Panamericana de la Salud 2007). Dentro de las enfermedades cardiovasculares se encuentra la hipertensión arterial (HTA) la cual es una de las principales causas de consulta y una de las enfermedades con más riesgo de desarrollar trastornos cardio-cerebrovasculares (Méndez \& Roxero 2007).

Por lo general esta patología es asintomática y se caracteriza por presión arterial sistólica (PAS) mayor a $139 \mathrm{mmHg}$ y/o diastólica (PAD) mayor a $89 \mathrm{mmHg}$ (Joint National Commitee 2007, Caja Costarricense de Seguro Social 2002). Su prevalencia se ha incrementado en todo el mundo entre un 3\% y un 18\% de 1999 a 2002 y 
su aparición puede estar relacionada con la presencia de factores de riesgo como: consumo de tabaco, dislipidemia, sedentarimo, estrés, sobrepeso, alcoholismo, dieta inadecuada, herencia, sexo y edad (Haijar \& Kotchen 2006).

En Costa Rica, solo se ha diagnosticado un $14 \%$ de la población con este padecimiento, pero al ser una condición a veces asintomática, es probable que el porcentaje sea mayor. Las tres primeras causas de mortalidad están asociadas con HTA (Enfermedad Isquémica Coronaria, Enfermedad Cerebrovascular y Enfermedad Hipertensiva). El sistema de salud ha mejorado la atención, pero no ha podido disminuir la mortalidad asociada con esta patología (Caja Costarricense de Seguro Social 2002).

La HTA es la segunda causa de consulta desde 1992, superada solo por las infecciones de la vías respiratorias superiores. Genera al menos 500000 consultas anuales y se prescriben entre tres y nueve medicamentos para su control, con altos costes económicos. Sin embargo, solo un pequeño porcentaje de la población diagnosticada se encuentra debidamente controlada, lo que significa que la intervención debe realizarse desde la óptica de otros factores que intervienen en su control (Comisión Interinstitucional Trazadora Hipertensión Arterial 2005).

La HTA puede ser controlada a través de múltiples factores resumidos en el término adherencia al tratamiento terapéutico, propuesto por la Organización Mundial de Salud (2003). La adherencia es la magnitud con que el paciente sigue las instrucciones médicas:

1. Toma el medicamento recetado apropiadamente

2. Cumple con las consultas de seguimiento

3. Ejecuta las modificaciones en los comportamientos sobre la dieta y estilo de vida

Una baja adherencia es causa importante de hipertensión arterial descontrolada; sin embargo, a veces no es reconocida y se le malinterpreta como resistencia al tratamiento farmacológico (Johnell et al. 2005)

La adherencia puede ser afectada por factores relacionados con la frecuencia de la dosis, las creencias sobre la salud, estatus socioeconómico, la depresión, el abuso, débil apoyo social y pobre relación entre paciente y médico. Al ser abordados a través de estrategias específicas, estos factores pueden facilitar la adherencia al tratamiento y disminuir el abandono (W Choo et al. 2001, Palacín et al. 2002, Bardorf et al. 2006, La Rosa et al. 2007).

El objetivo de la adherencia no es solo controlar las cifras de tensión arterial, sino también brindar años con buena calidad de vida y evitar las posibles complicaciones de esta patología. El tratamiento médico debe ir acompañado de recomendaciones como disminuir el consumo de sodio, evitar el fumado y el alcohol, controlar el estrés, hacer ejercicio y mantener un peso adecuado (Bautista \& Pérez 2003, Holguín et al. 2008, Rivas et al. 2008).

El éxito del tratamiento no solo implica el tomarse el medicamento sino un compromiso eficaz ante sus beneficios (Crespo et al. 2000, Holguín et al. 2008).

Tras revisar toda la bibliografía disponible, creemos que este trabajo presenta el primer estudio sobre la relación entre la adherencia al tratamiento antihipertensivo y el sexo, condiciones socio-demográficas y la relación con el médico en el personal de una universidad a distancia.

\section{METODOLOGÍA}

\section{Instrumentos}

Los datos se obtuvieron el expediente médico y un cuestionario: el Cuestionario de Evaluación de Adherencia Terapéutica en pacientes hipertensos con 36 ítemes sobre antecedentes personales, médicos, de adherencia y del entorno. Este cuestionario está adaptado de la Prueba de Morinsky y del cuestionario Martin-Bayarre-Grau o "MBG". Ambas pruebas fuente han sido validadas por expertos y utilizados en los estudios dirigidos en esta línea de investigación (García et al. 2000, Martín et al. 2008). La Prueba de Morinsky es un método de evaluación indirecta del cumplimiento terapéutico y es considerado altamente fiable por estar basado en el autoinforme. El cuestionario MBG está conformado por 12 ítemes de la Escala Lickert que presenta cinco posibilidades de respuesta desde "siempre" hasta "nunca" (García et al. 2000, Martín et al. 2008).

\section{Análisis estadístico}

Usamos los programas Excel y SPSS 16.0 para agrupar y analizar los datos. Para las pruebas de hipótesis usamos Chi-cuadrado de Contingencia, Prueba Exacta de Fisher y U de Mann- Whitney.

\section{Población}

Se convocó a los 179 pacientes hipertensos, que corresponde al total de la población hipertensa de la universidad. Únicamente 80 respondieron el cuestionario.

Todos los pacientes firmaron el consentimiento informado y el estudio cuenta con la aprobación Ética de la Vicerrectoría de Investigación de la UNED. La documentación correspondiente queda archivada en el Consultorio Médico de la UNED. 


\section{Procedimientos}

La recolección de los datos se realizó en dos etapas: la primera fue la revisión del expediente médico (total 1526 expedientes), para determinar cuáles pacientes habían consultado por HTA. La segunda etapa fue la convocatoria vía correo electrónico a la población hipertensa (179 personas) para la aplicación del cuestionario, el cual fue respondido por 80 pacientes.

\section{RESULTADOS}

\section{Características en que hay diferencia entre mujeres y hombres}

\section{Características personales}

Las variables cuantitativas se presentan en el formato: Promedio $(X) \pm$ Desviación Estándar; Valor Mínimo - Valor Máximo; Tamaño de Muestra.

Los hombres son más pesados $(X=84,78 \pm 13,68 \mathrm{DE}$; $63-120 \mathrm{~kg} ; \mathrm{N}=41)$ que las mujeres $(X=73,72 \pm 12,14 \mathrm{DE}$; 52 - 110kg; $N=39 ;$ U Mann-Whitney $=415,500 ; p=0,000$ ) y son en promedio más altos (Hombres $X=1,71 \pm 0,77$ $\mathrm{DE} ; 1,60-1,90 \mathrm{~m} ; \mathrm{N}=41$. Mujeres: $X=1,59 \pm 0,05 \mathrm{DE} ; 1,49$ $-1,75 \mathrm{~m} ; \mathrm{N}=39 . ; \mathrm{U}$ Mann-Whitney $=157,000 ; p=0,000$ ).

\section{Control de la enfermedad}

La mayoría de las mujeres afirmaron realizar el control arterial "Siempre" o "A veces", mientras que la mayoría de los hombres respondieron "Siempre" o "Casi Siempre" (Cuadro 1; Chi-cuadrada $=1,332 ; p=0,021$ ).

\section{CUADRO 1}

Relación entre realizar el control arterial y género de pacientes hipertensos, UNED, 2009

\begin{tabular}{lcc}
\hline Control arterial & Mujeres $(\mathbf{N}=\mathbf{3 9})$ & Hombres $(\mathbf{N}=\mathbf{4 1})$ \\
\hline Siempre & 69,2 & 43,9 \\
Casi siempre & 2,6 & 34,1 \\
A veces & 15,4 & 12,2 \\
Casi nunca & 2,6 & 2,4 \\
Nunca & 7,7 & 4,9 \\
No aplica & 2,6 & 2,4
\end{tabular}

La mayoría de los hombres manifestaron hacer ejercicio "A veces", seguido de "Siempre" o "Casi Siempre", mientras que las mujeres se repartieron de forma más homogénea en todas las categorías desde "Siempre" hasta "Casi Nunca" (Cuadro 2; Chi-cuadrada $=1,170 ; p=0,039$ ).

\section{Percepción del médico}

Un alto porcentaje de los 41 hombres entrevistados $(87,8 \%)$ percibe que el médico explica con claridad el modo de seguir el tratamiento; en el caso de las mujeres, sólo un $64,1 \%$ de las 39 entrevistadas tiene esa percepción (Prueba Exacta de Fisher, $p=0,012$ ).

\section{CUADRO 2}

Relación entre la ejecución de los ejercicios físicos indicados y género de pacientes hipertensos, UNED, 2009

\begin{tabular}{lcc}
\hline $\begin{array}{l}\text { Frecuencia con que } \\
\text { hace ejercicio }\end{array}$ & Mujeres $(\mathrm{N}=39)$ & Hombres $(\mathrm{N}=41)$ \\
\hline Siempre & 23,1 & 29,3 \\
Casi siempre & 15,4 & 17,1 \\
A veces & 20,5 & 41,5 \\
Casi nunca & 23,1 & 7,3 \\
Nunca & 12,8 & 0 \\
No aplica & 5,1 & 4,9
\end{tabular}

\section{Características en que no hay diferencia entre mujeres y hombres}

\section{Características personales}

En el Cuadro 3 se presentan los datos para edad, índice de masa corporal (IMC), estado civil, nivel educativo y ocupación, aspectos para los cuales, no existe diferencia estadísticamente significativa entre géneros.

Dichos datos se presentan agrupados y en cada caso se incluye la prueba estadística correspondiente. En general, el ámbito de edades es amplio y se puede observar que la mayoría de los pacientes son casados, poseen estudios universitarios completos y se desempeñan como profesionales.

\section{Hábitat}

La mayoría señala que en su casa cuentan con mucho espacio y suficientes zonas verdes. En su barrio el ruido de 
CUADRO 3

Características socio-demográficas (\%) según género de pacientes hipertensos, UNED, 2009

\begin{tabular}{|c|c|c|}
\hline & Mujeres ( $\mathrm{N}=39)$ & Hombres $(\mathrm{N}=41)$ \\
\hline \multicolumn{3}{|c|}{ Edad $(U$ Mann-Whitney $=609,500 ; p=0,067)$} \\
\hline & $\begin{array}{c}X=51,31 \pm 7,11 \mathrm{DE} \\
35-74 \text { años }\end{array}$ & $\begin{array}{c}X=47,46 \pm 9,86 \mathrm{DE} ; \\
22-66 \text { años }\end{array}$ \\
\hline \multicolumn{3}{|c|}{ IMC (U Mann-Whitney $=766500 ; p=0,715)$} \\
\hline & $\begin{array}{c}X=29,03 \pm 5,20 \mathrm{DE} \\
20,82-47,61 \mathrm{~kg} / \mathrm{m}^{2}\end{array}$ & $\begin{array}{l}X=28,74 \pm 3,59 \mathrm{DE} ; \\
20,05-34,78 \mathrm{~kg} / \mathrm{m}^{2}\end{array}$ \\
\hline \multicolumn{3}{|c|}{ Estado civil (Chi-cuadrada $=4,225 ; p=0,376$ ) } \\
\hline Soltero/a & 25,6 & 24,4 \\
\hline Casado/a & 46,2 & 63,4 \\
\hline Divorciado/a & 17,9 & 9,8 \\
\hline Viudo/a & 2,6 & 0 \\
\hline Unión libre & 7,7 & 2,4 \\
\hline \multicolumn{3}{|c|}{ Nivel educativo (Chi-cuadrada $=3,410 ; p=0,333$ ) } \\
\hline Primaria completa & 0 & 0 \\
\hline Primaria incompleta & 0 & 0 \\
\hline Secundaria completa & 12,8 & 7,3 \\
\hline Secundaria incompleta & 5,1 & 0 \\
\hline Universitaria completa & 61,5 & 75,6 \\
\hline Universitaria incompleta & 20,5 & 17,1 \\
\hline \multicolumn{3}{|c|}{ Ocupación (Chi-cuadrada $=8,561 ; p=0,073$ ) } \\
\hline Administrativo/a & 46,2 & 22,0 \\
\hline Profesional & 43,6 & 65,9 \\
\hline Pre-universitario/a & 0 & 0 \\
\hline Técnico/a & 0 & 4,9 \\
\hline Personal de apoyo & 2,6 & 4,9 \\
\hline Pensionado/a & 7,7 & 2,4 \\
\hline
\end{tabular}

los vehículos y los problemas de criminalidad son pocos. Además, admiten que no participan en actividades deportivas o recreativas (Cuadro 4).

\section{Percepción del médico}

La mayoría indica que el médico les explica con claridad la importancia de seguir el problema de la hipertensión, el tratamiento a seguir y la importancia de seguir las indicaciones cuidadosamente. Pocos indicaron que el médico no les explica nada con claridad (Cuadro 5).

\section{Seguimiento del tratamiento}

La mayoría manifestaron ingerir los medicamentos en el horario establecido, tomar las dosis indicadas por el médico y cumplir las indicaciones relacionadas con la dieta.

También dijeron asistir a las consultas de seguimiento, cumplir con el tratamiento sin necesidad de grandes esfuerzos y sin la supervisión de su familia o amigos.

La mayoría manifestaron que "Nunca" dejan de tomar el tratamiento cuando se sienten bien (Cuadro 6). 


\section{CUADRO 4}

Características del entorno(\%) de pacientes hipertensos, según género, UNED, 2009

Mujeres ( $\mathrm{N}=39) \quad$ Hombres $(\mathrm{N}=41)$

\begin{tabular}{|c|c|c|}
\hline \multicolumn{3}{|l|}{$\begin{array}{l}\text { Espacio que disfrutan en casa } \\
\text { (Chi-cuadrada }=1,280 ; p=0,527 \text { ) }\end{array}$} \\
\hline Mucho espacio, se mueve libremente & 66,7 & 73,2 \\
\hline Espacio mediano & 30,8 & 26,8 \\
\hline Muy poco espacio & 2,6 & 0 \\
\hline \multicolumn{3}{|l|}{$\begin{array}{l}\text { Problemas de criminalidad en el barrio } \\
\text { (Chi-cuadrada }=4,303 ; p=0,116)\end{array}$} \\
\hline Pocos & 74,4 & 73,2 \\
\hline Medianos & 12,8 & 24,4 \\
\hline Grandes & 12,8 & 2,4 \\
\hline \multicolumn{3}{|l|}{$\begin{array}{l}\text { Participación en actividades recreativas } \\
\text { (Prueba Exacta de Fisher; } p=0,586 \text { ) }\end{array}$} \\
\hline Sí & 48,7 & 48,8 \\
\hline No & 51,3 & 52,1 \\
\hline \multicolumn{3}{|c|}{$\begin{array}{l}\text { Ruido de vehículos percibido en el barrio } \\
\text { (Chi-cuadrada }=3,652 ; p=0,161 \text { ) }\end{array}$} \\
\hline Poco & 53,8 & 58,5 \\
\hline Normal & 46,2 & 34,1 \\
\hline Excesivo & 0 & 7,3 \\
\hline \multicolumn{3}{|l|}{$\begin{array}{l}\text { Cantidad de zonas verdes donde habita } \\
\text { (Chi-cuadrada }=1,231 ; p=0,540 \text { ) }\end{array}$} \\
\hline Pocas o nulas & 33,3 & 34,1 \\
\hline Suficientes & 51,3 & 41,5 \\
\hline Abundantes, tengo plantas a la vista & 15,4 & 24,4 \\
\hline
\end{tabular}

CUADRO 5

Tipo de explicaciones que reciben los pacientes hipertensos de sus médicos (\%), según género, UNED, 2009

\begin{tabular}{lcc}
\hline Explicación que da el médico & Mujeres (N=39) & Hombres (N=41) \\
\hline $\begin{array}{l}\text { Importancia del tratamiento } \\
\text { (Prueba Exacta de Fisher; } \mathrm{p}=0,327)\end{array}$ & 87,2 & 92,7 \\
$\begin{array}{l}\text { Problema de hipertensión } \\
\text { (Prueba Exacta de Fisher; } \mathrm{p}=0,091)\end{array}$ & 56,4 & 73,2 \\
$\begin{array}{l}\text { No explica nada } \\
\text { (Prueba Exacta de Fisher; } \mathrm{p}=0,615)\end{array}$ & 10,3 & 9,8 \\
$\begin{array}{l}\text { Importancia de tomar el tratamiento } \\
\text { (Prueba Exacta de Fisher; } \mathrm{p}=0,481)\end{array}$ & 97,4 & 100
\end{tabular}


CUADRO 6

Características relativas al cumplimiento terapéutico (\%), según género, UNED, 2009

\begin{tabular}{|c|c|c|c|c|c|c|}
\hline & Siempre & Casi siempre & $A$ veces & Casi nunca & Nunca & No aplica \\
\hline \multicolumn{7}{|c|}{ Percepción de la ingesta de medicamentos en el horario establecido (Chi-cuadrada =0,300; $p=0,861$ ) } \\
\hline Hombres $(\mathrm{N}=41)$ & 75,6 & 19,5 & 4,6 & 0 & 0 & 0 \\
\hline Mujeres $(\mathrm{N}=39)$ & 76,9 & 20,5 & 2,6 & 0 & 0 & 0 \\
\hline \multicolumn{7}{|c|}{ Toma de las dosis indicadas por el médico (Chi-cuadrada $=1,167 ; p=0,558$ ) } \\
\hline Hombres $(\mathrm{N}=41)$ & 95,1 & 2,4 & 2,4 & 0 & 0 & 0 \\
\hline Mujeres $(\mathrm{N}=39)$ & 89,7 & 7,7 & 2,6 & 0 & 0 & 0 \\
\hline \multicolumn{7}{|c|}{ Cumplimiento de las indicaciones relacionadas con la dieta (Chi-cuadrada $=3,657 ; p=0,600$ ) } \\
\hline Hombres $(\mathrm{N}=41)$ & 22,0 & 36,6 & 26,8 & 7,3 & 0 & 7,3 \\
\hline Mujeres $(\mathrm{N}=39)$ & 20,5 & 41,0 & 15,4 & 7,7 & 5,1 & 10,3 \\
\hline \multicolumn{7}{|c|}{ Asistencia a las consultas de seguimiento (Chi-cuadrada $=8,233 ; p=0,144$ ) } \\
\hline Hombres $(\mathrm{N}=41)$ & 53,7 & 14,6 & 17,1 & 7,3 & 0 & 7,3 \\
\hline Mujeres $(\mathrm{N}=39)$ & 61,5 & 12,8 & 2,6 & 2,6 & 2,6 & 17,9 \\
\hline \multicolumn{7}{|c|}{ Cumplimiento del tratamiento sin supervisión (Chi-cuadrada $=5,009 ; p=0,286$ ) } \\
\hline Hombres $(\mathrm{N}=41)$ & 85,4 & 7,3 & 2,4 & 0 & 4,9 & 0 \\
\hline Mujeres $(\mathrm{N}=39)$ & 94,9 & 2,6 & 0 & 0 & 0 & 2,6 \\
\hline \multicolumn{7}{|c|}{ Cumplimiento del tratamiento sin grandes esfuerzos (Chi-cuadrada $=0,699 ; p=0,705$ ) } \\
\hline Hombres $(\mathrm{N}=41)$ & 80,5 & 14,6 & 0 & 0 & 4,9 & 0 \\
\hline Mujeres $(\mathrm{N}=39)$ & 87,2 & 10,3 & 0 & 0 & 2,6 & 0 \\
\hline \multicolumn{7}{|c|}{ Deja de tomar el tratamiento cuando se siente bien (Chi-cuadrada $=8,641 ; p=0,124$ ) } \\
\hline Hombres $(\mathrm{N}=41)$ & 7,3 & 7,3 & 4,9 & 17,1 & 58,5 & 4,9 \\
\hline Mujeres (N=39) & 10,3 & 0 & 7,7 & 2,6 & 69,2 & 10,3 \\
\hline
\end{tabular}

\section{DISCUSIÓN}

\section{Características donde hubo diferencias entre hombres y mujeres}

\section{Características personales}

El peso y la talla son dos medidas de gran importancia para poder determinar el índice de masa corporal o IMC, sin embargo, aunque el IMC es ampliamente usado para evaluar el estado nutricional de la población, no es un factor determinante porque no permite conocer el índice de grasa. Como era de esperarse, en este estudio los hombres son más altos y pesados, sin embargo esto no influyó en el IMC, al igual que en poblaciones previamente estudiadas (Johnell et al. 2005). A pesar de que la adherencia de los pacientes es buena y los pacientes afirmaron recibir recomendaciones alimenticias, el IMC se encuentra por arriba del $25 \%$ recomendado en ambos grupos. Pareciera que poseen los conocimientos pero no los aplican en su dieta diaria. Esto podría generar a largo plazo un factor concomitante para el desarrollo de otras patologías asociadas a sobrepeso e hipertensión, tal como el síndrome metabólico. 


\section{Control de la enfermedad}

Tanto los hombres como las mujeres realizan un control mensual de la medición de presión arterial y algún tipo de actividad física recomendada, sin embargo los hombres reportaron algo más de ejercicio. Esto puede deberse a que las mujeres priorizan otras cosas como el trabajo en la casa, el empleo y los hijos, en lugar del autocuidado de salud (Weisner et al. 2006). Tradicionalmente se ha considerado el sexo femenino como figura cuidadora, capaz de sacrificar su salud en pro de los demás; la carga laboral y familiar pareciera ocupar los espacios en los que las mujeres podrían dedicarse a otras actividades entre ellas el realizar ejercicios fuera o dentro de su casa, no así en los hombres quienes por lo general colaboran poco o nada con las cargas familiares.

\section{Percepción del médico}

Más hombres percibieron como adecuadas las explicaciones médicas sobre cómo seguir el tratamiento. El uso de la jerga médica hace que los pacientes perciban un distanciamiento y esto podría ser más marcado en las mujeres (Hall et al. 2007). Tal vez los hombres acuden menos al médico y por lo general preguntan poco acerca de las dolencias que los llevaron a consultar. La percepción del médico es de vital importancia para la adherencia a cualquier tratamiento. En este caso, las mujeres perciben un distanciamiento por parte del personal médico (que en la UNED es todo femenino), pero muestra diferencias significativas en la adherencia con respecto a los hombres.

\section{Características en que no hay diferencia entre mu- jeres y hombres}

\section{Características personales}

La edad de aparición de HTA es mayor en hombres después de los 30 años y se iguala a partir de los 50 años entre hombres y mujeres, debido a la disminución de hormonas femeninas (Suárez \& Gabriel 2000). Pero en nuestro caso no hubo diferencias por edad e IMC, tal vez porque la población sea relativamente homogénea en cuanto a las características y factores de riesgo como sedentarismo, nivel educacional, ocupación y nivel de estrés (Espinoza et al. 2004).

\section{Hábitat}

El espacio físico, las áreas de recreación, el entorno social (ruido, criminalidad y drogas), además de la realización de actividades recreativas, son características similares en ambos sexos, lo cual puede explicar que no se hallaran efectos de estos factores que sí son conocidos como de importancia, por ejemplo en estudios realizados en Holanda, donde se pudo determinar que los factores del entorno social son contundentes en la aparición de HTA (Agyemang et al. 2007).

\section{Percepción del médico}

La percepción que tiene un paciente hipertenso de su médico define en gran medida su adherencia al tratamiento, la satisfacción del paciente y la eficacia del control terapéutico (Ramírez et al. 1998). En la UNED, los pacientes se muestran satisfechos con el servicio médico en cuanto a la explicación, diagnóstico, control terapéutico y seguimiento; por lo que es de esperar que su adherencia al tratamiento sea buena.

\section{Conclusiones generales}

Las características socio-demográficas no presentan diferencias significativas en cuanto al género y su adherencia al tratamiento.

Existe la posibilidad de que los factores psicológicos y sociales intervengan en una adherencia adecuada al tratamiento, estos no se contemplaron en este primer estudio.

Los pacientes refieren altos conocimientos en cuanto a medidas de control de la hipertensión como lo son: dieta, ejercicio, control médico y la importancia del tratamiento.

No se pudo determinar la relación entre las actividades recreativas, ruido y espacio físico en la aparición de HTA, pues los resultados fueron homógeneos en la población estudiada. Sin embargo se ha demostrado que en otros casos están altamente relacionados con la HTA.

La percepción del personal médico es satisfactoria y aparentemente esto permite un mayor control terapéutico de esta patología.

\section{REFERENCIAS}

Agyemang, C., C. van Hooijdonk, W. Wendel-Vos, J. Ujcic-Voortman, E. Lindeman, K. Stronks \& M. Droomers. 2007. Ethnic differences in the effect of environmental stressors on blood pressure and hypertension in the Netherlands. BMC Public Health 7-118.

Bardorf, T., F. Hecht, C. Rubow \& J. Gerstoft. 2006. Physicians' communication with patients about adherence to HIV medication in San Francisco and Copenhagen: a qualitative study using Grounded Theory. BioMed Central Services Research 6: 1-12.

Bautista, A. \& G. Pérez. 2003. Evaluación y manejo actual de la hipertensión arterial esencial. Acta Neurológica Colombia 19: 69-72. 
Caja Costarricense de Seguro Social. 2002. Estadística de los Servicios de Salud, 2002. Departamento de Estadística. Costa Rica.

Caja Costarricense de Seguro Social. 2002. Guías para la detección, diagnóstico y tratamiento de la Hipertensión Arterial en el primer nivel de atención. Costa Rica, 2-62.

Caja Costarricense de Seguro Social, Ministerio de Salud y Organización Panamericana de la Salud. Comisión Interinstitucional Trazadora Hipertensión Arterial. (2005). Metodología para evaluar la calidad de la atención de la hipertensión arterial: trazadora de hipertensión arterial. Costa Rica, 1-96.

Crespo, N., J. Rubio, M. Casado, J. Sánchez \& S. Campo. 2000. Factores modulares de la adherencia farmacológica en hipertensión arterial, estudio exploratorio. Rev. Hipertensión España 17: 1-7.

Espinoza, F., G. Ceja, B. Trujillo, T. Uribe, P. Abarca \& D. Flores. 2004. Análisis de los factores de riesgo de la hipertensión arterial en Colima, México. Rev Panamá Salud Pública 16: 402-407.

García A., F. Leiva, F. Martos, A. García \& D. Prados. 2000. ¿Cómo diagnosticar el cumplimiento terapéutico en atención primaria? Medicina de la Familia 1: 13-19.

Haijar, I. \& T. Kotchen. 2006. Hypertension: Trends in prevalence, incidence and control. Annual Review Public Health 27:465-490.

Hall, R., J. Hernández, O. Carranza, F. Serrano, J. Martínez, G. Rosencranc \& L. St. Clai. 2007. Relaciones médico- paciente, un estudio empírico. Medicina y Ética, 3, 315-336.

Holguín, L., D. Correa, M. Aivillaga, M. Cáceres \& M. Varela. 2006. Adherencia al tratamiento de Hipertensión Arterial: Efectividad de un programa de intervención biopsicosocial. University Psychologic Colombia 5: 535-547.

Johnell, K., L. Råstam, T. Lithman, \& J. Merlo. 2005. Low adherence with antihypertensives in actual practice: the association with social participation - a multilevel analysis. BioMed Central Public Health 5:1-7.

Joint National Committee. 2007. The Seventh Report of the Joint National Committee on Prevention, Detection, Evaluation, and Treatment of High Blood Pressure. National Heart
Lung and Blood Institute.

La Rosa, Y., L. Martín \& H. Bayarre. 2007. Adherencia terapéutica y factores psicosociales en pacientes hipertensos. Rev. Cubana Medicina Gral Integral 23:1-5.

Martín, L., H. Bayarre \& J. Grau. 2008. Validación del cuestionario MBG (Martín-Bayarre-Grau) para evaluar la adherencia terapéutica en hipertensión arterial. Rev. Cubana Salud Pública 34.

Méndez, E. \& L. Roxero. 2007. Prevalencia de Hipertensión en adultos mayores en Costa Rica. Población salud Mesoamérica 5: 1-9.

Organización Mundial de Salud. 2003. Adherencia a los tratamientos a largo plazo, pruebas para la acción 1-187.

Organización Panamericana de la Salud. 2007. Encuesta de Diabetes, Hipertensión y factores de riesgo de enfermedades crónicas. Guatemala: PAHO 1-77.

Palacín, F., C. Silva \& L. Krotter. 2002. Factores cognitivos en la adherencia al tratamiento. Congreso Internacional de Psiquiatría. Argentina.

Ramírez, T., P. Nájera \& G. Nigenda. 1998. Percepción de la calidad de la atención de los servicios de salud en México: Perspectiva de los usuarios. Rev. Salud Pública México 40: 1-5.

Rivas, J., C. Gutiérrez \& J. Rivas. 2008. Tratamiento y costos farmacológicos de la Hipertensión Arterial no complicada en un hospital regional. Rev. Peruana Cardiología 34: 181-190.

Suárez, C. \& R. Gabriel. 2000. Epidemiología de la Hipertensión Arterial en España. Cardiovascular Risk Factors 9: 239246.

W Choo, P., C. Rand, T. Inui, M. Ting Lee, C. Canning \& R. Platt. 2001. A cohort study of possible risk factors for over-reporting of antihypertensive adherence. BioMed Central Cardiovasculars Disorders 1: 1-6.

Weisner, C., V. Vejarano, M. Caicedo, M. Tovar \& D. Cendales. 2006. La citología de cuello uterino en Soacha, Colombia, representaciones sociales, barreras y motivaciones. Rev. Salud Pública Univ. Nacional Colombia 8:185-196. 